\title{
INOVAÇÃO E DESENVOLVIMENTO DE PRODUTOS NA INDÚSTRIA DE COSMÉTICOS: UM ESTUDO DE CASO
}

\section{INNOVATION AND PRODUCTS DEVELOPMENT IN THE COSMETIC INDUSTRY: A CASE STUDY}

\author{
Ana Cristina Avelar ${ }^{1}$; Cristina Gomes de Souza ${ }^{2}$ \\ ${ }^{1}$ Ana Cristina Avelar - CEFET-RJ - Rio de Janeiro - Brasil - anacrisavelar@yahoo.com.br \\ ${ }^{2}$ Cristina Gomes de Souza - CEFET-RJ - Rio de Janeiro - Brasil - cgsouza@,cefet-rj.br
}

\begin{abstract}
Resumo
Pode-se dizer que dentre as quatro variáveis relacionadas ao desenvolvimento - terra, capital, trabalho e tecnologia -, a tecnologia é a que mais peso tem na determinação da capacidade competitiva de um setor. Atualmente com o nivelamento das empresas quanto aos aspectos de qualidade e produtividade, a gestão da tecnologia passou a despontar como importante diferencial de competitividade. A importância da inovação tecnológica para a competitividade é inquestionável, seja para ganhar mercados através da introdução de novos produtos e processos ou produzir com máximo aproveitamento físico de insumos a fim de reduzir custos. Dentro desse contexto, o desenvolvimento de novos processos e produtos, está se tornando uma função primordial para as empresas. A indústria de cosméticos tem apresentado expressivo crescimento nos últimos anos com um aumento no seu faturamento de 19\% no ano de 2004 em comparação a 2003. Esse crescimento do setor reflete uma competitividade acirrada exigindo das empresas o lançamento constante de novos produtos bem como reformulações de embalagens. Para acompanhar esse dinamismo torna-se necessário a adoção de estratégias voltadas para a gestão da inovação e do processo de desenvolvimento de produto. O objetivo do trabalho é apresentar um estudo de caso numa empresa do setor a fim de verificar como se dá o processo de desenvolvimento do produto em relação aos modelos existentes na literatura. Estes modelos possuem como ponto de partida o estabelecimento dos objetivos mercadológicos para a empresa, que busca nas necessidades do consumidor e oportunidades de mercado o foco para o desenvolvimento de novos produtos.
\end{abstract}

Palavras-Chave: inovação tecnológica, indústria de cosméticos, desenvolvimento de novos produtos

\section{Introdução}

A nova competição industrial - que apresenta um de seus focos no processo de desenvolvimento de produtos - considera três forças que surgiram nas empresas nas duas ultimas décadas: uma competição internacional intensa proveniente da globalização; consumidores mais sofisticados provenientes da fragmentação dos mercados; e mudanças constantes na tecnologia. 
Essas três forças combinaram-se levando o processo de desenvolvimento de produtos ao centro do jogo de competição entre as empresas (CLARK \& FUJIMOTO, 1991).

A indústria de cosméticos engloba os setores de higiene pessoal, perfumaria e cosméticos e, conforme Garcia e Furtado (2002) caracteriza-se pela necessidade contínua de pesquisas de novos insumos e introdução de inovações em suas linhas de produtos. Este fato pode ser considerado um fator de importância para a competitividade no setor. Existe uma heterogeneidade nesta indústria, pois a presença de grandes empresas internacionais, diversificadas ou especializadas nos segmentos de perfumaria e cosméticos, é contrastada com um grande número de pequenas e médias empresas com atuação focalizada na produção de cosméticos.

Verifica-se que as grandes empresas do setor e, principalmente, as empresas transnacionais adotam estratégias definidas com relação ao desenvolvimento de novos produtos investindo em centros próprios de Pesquisa e Desenvolvimento (P\&D) e/ou parcerias extra-muros e com processos de gestão do conhecimento estruturados. Já as pequenas e médias empresas muitas vezes não dispõem de capital necessário para a realização de investimentos de $\mathrm{P} \& \mathrm{D}$ ou de uma situação econômico-financeira que permita assumir os riscos inerentes ao desenvolvimento de novos produtos. Muitas dessas empresas também não dispõem de uma gestão do conhecimento que remeta a processos de desenvolvimento de produtos realmente sistematizados.

No Brasil, embora seja predominante a presença das grandes empresas internacionais do setor, pode-se observar a existência de um grande número de empresas de capital nacional, algumas das quais vem realizando atividades de desenvolvimento de produto no país, adotando estratégias no sentido de incrementar sua participação no mercado nacional e também no mercado internacional.

O objetivo desse trabalho é apresentar um estudo de caso sobre o desenvolvimento de produto em uma empresa nacional de médio porte do setor de cosméticos do estado do Rio de Janeiro. Para tanto foi feita uma pesquisa de campo com realização de visitas técnicas e entrevistas na empresa em questão. A partir da análise realizada pretende-se verificar: Como o desenvolvimento de novos produtos é inserido na estratégia da empresa; como é realizado, ou seja, quais as etapas que compõem o processo de desenvolvimento de produtos; e se existe um processo estruturado para tal, isto é, se segue algum determinado modelo de processo de desenvolvimento de produto encontrado na literatura específica sobre o assunto.Como modelos de desenvolvimento de produto utilizados para análise estão os modelos seqüencial, flexível, integrativo e improvisacional, analisados por Cunha e Gomes em artigo publicado em 2003, e o modelo de Fuller apresentado em 1994. 


\section{Inovação e Desenvolvimento de Produtos na Estratégia das Empresas}

De uma forma ampla, deve-se considerar inovação em uma empresa não somente as inovações com base tecnológica, mas também as de qualquer outro tipo, como inovações em marketing, distribuição, em processos administrativos e organizacionais que ajudem a obter vantagens competitivas. O importante é que o processo de inovação dentro de uma empresa não pode estar separado do seu contexto estratégico e competitivo (PORTER, 1990 apud MONTANA, 2001). Para Fleury (1999), as mudanças nas condições de competição, a globalização dos mercados e da produção e o advento da economia do conhecimento são fatores que vêm impactando as novas estratégias competitivas das empresas transnacionais que lideram o processo de globalização, em especial em relação ao papel que reserva às suas operações em países emergentes.

Ao analisar a questão das estratégias das empresas neste novo cenário mundial, Mintzberg e Quinn (2001) consideram que existe uma necessidade constante das empresas de estar aperfeiçoando o seu negócio. Este processo de aperfeiçoamento dos negócios pode ser feito através do desenvolvimento de seus produtos dentro de sua estrutura, desenvolvimento de seu mercado via novos segmentos, novos canais ou novas áreas geográficas ou a colocação dos mesmos produtos de maneira mais vigorosa para os mesmos mercados.

Neste processo verifica-se que a estratégia de desenvolvimento de produto abrange desde uma estratégia simples de extensão de produto, até uma mais abrangente, de proliferação da linha de produto ou do inverso de racionalização de linha de produtos. Pode ser utilizada a estratégia de oferecer produtos novos e modificados no mesmo negócio básico. Se na estratégia de extensão de produto esses forem novos e distintos, será utilizada a diferenciação por design, porém se for o acréscimo à linha de produtos padronizados, será feita a diferenciação através da segmentação de mercado. A proliferação da linha de produtos significa partir em direção da segmentação abrangente de produtos cobrindo completamente um determinado mercado. A racionalização significa escolher determinados produtos e diminuir a linha de produtos para eliminar produtos sobrepostos e excessos não-lucrativos.

Segundo Baxter (2000) existem quatro tipos de estratégias de inovação e desenvolvimento de produto. São as estratégias ofensivas, defensivas, tradicionais e dependentes. As empresas que adotam a estratégia ofensiva são aquelas que querem estar sempre à frente de seus concorrentes e investem pesadamente em pesquisa e desenvolvimento para introduzir inovações radicais ou incrementais em seus produtos. É uma estratégia pró-ativa e trabalha com a perspectiva de longo prazo para o retorno dos investimentos. A estratégia defensiva é usada por empresas que querem seguir as empresas líderes sem arcar com os custos maiores de desenvolvimento e com o risco de criar novos mercados. Esta estratégia depende da rapidez com que a empresa consegue absorver as 
inovações lançadas pelos concorrentes e introduzir melhorias nestes produtos. Apesar de ter menores custos e menores riscos esta estratégia também dá menor lucratividade.

As empresas que utilizam a estratégia tradicional atuam em mercados estáveis com pouca demanda para mudanças e com uma linha de produtos estáticos. As inovações são pequenas limitando-se a mudanças mínimas no produto para reduzir custos, facilitar a produção ou aumentar a confiabilidade do produto. A estratégia dependente é adotada por empresas que não tenham autonomia para lançar novos produtos, pois dependem de autorização de suas matrizes ou então de seus clientes para a introdução de inovações. São empresas que são subsidiárias de outras ou então trabalham sob encomenda. As inovações geralmente se limitam às melhorias de processo.

Qual estratégia será usada irá depender do planejamento estratégico da empresa, se ela é uma empresa inovadora e em que grau isto acontece, pois o desenvolvimento de produtos pode variar desde uma inovação radical até uma cópia completa do concorrente. Decidir em qual ponta colocar a empresa é uma questão de risco gerencial, pois de um lado existe o risco máximo de arcar com o desenvolvimento pioneiro e o produto não ser aceito pelo mercado. Do outro lado a cópia de produtos pode resultar em problemas judiciais com a questão de marcas e patentes ou o fracasso de mercado por falta de diferenciação.

\section{Modelos de Desenvolvimento de Produtos}

Cunha e Gomes (2003) analisam o desenvolvimento de produto através de cinco modelos básicos que são os modelos seqüencial, compressão, flexível, integrativo e improvisacional. Estes seriam escolhidos dependendo das necessidades e características do projeto e do mercado e da velocidade de assimilação de novas tecnologias (Tabela 1).

O modelo seqüencial confia particularmente no planejamento, na antecipação e no controle. É um processo que trabalha com a expectativa de redução do risco inerente da inovação sugerindo a quantidade de etapas e a seqüência que será feita e constitui a perspectiva dominante na gerência da inovação de produto. São apresentados como marcos para projetos seguros e eficientes de desenvolvimento de produto novo. Entre as etapas, existem momentos de decisão onde se verifica se o processo deve continuar ou ser interrompido. É um modelo de controle e padronização da inovação do produto retirando a criatividade do processo. Esta característica pode fazê-los mais apropriados para as inovações incrementais, do que para inovações radicais.

O modelo de compressão pode ser pensado como uma versão do modelo seqüencial ajustado aos ambientes de mudanças rápidas. Fatos como pressões do mercado, ajustes dos ciclos de vida do produto, e da importância do tempo de lançamento fazem com que algumas etapas necessitem às vezes serem aceleradas ou encurtadas. Existem várias maneiras de conseguir a 
compressão seja melhorando o planejamento, simplificando o processo, eliminando etapas desnecessárias, envolvendo fornecedores, encurtando a época da conclusão de cada etapa ou etapas se sobrepondo. Assim, a lógica é que algumas tarefas do processo possam começar simultaneamente, em vez de seguir uma seqüência rígida de etapas de desenvolvimento. Este modelo presume que as atividades de desenvolvimento podem ser conhecidas com antecedência e que os modelos de inovação de produtos devem reduzir as incertezas o máximo possível.

Tabela 1 - Modelos de desenvolvimento de produtos

\begin{tabular}{|c|c|c|c|}
\hline $\begin{array}{l}\text { Modelo de } \\
\text { inovação de } \\
\text { produto }\end{array}$ & Hipótese & Objetivo principal & $\begin{array}{l}\text { Estrutura do } \\
\text { processo }\end{array}$ \\
\hline Seqüencial & $\begin{array}{l}\text { Certeza, equilíbrio, } \\
\text { premeditável, } \\
\text { estabilidade, } \\
\text { mecanicista. }\end{array}$ & Padronizar a inovação & $\begin{array}{l}\text { Universal: Todos os } \\
\text { produtos; todas as } \\
\text { organizações, todas } \\
\text { as indústrias. }\end{array}$ \\
\hline Compressão & $\begin{array}{l}\text { Certeza, equilíbrio, } \\
\text { estabilidade, } \\
\text { adaptação, } \\
\text { mecanicista. }\end{array}$ & $\begin{array}{l}\text { Otimizar o modelo } \\
\text { seqüencial }\end{array}$ & $\begin{array}{l}\text { Universal: Todos os } \\
\text { produtos; todas as } \\
\text { organizações, todas } \\
\text { as indústrias. }\end{array}$ \\
\hline Flexível & $\begin{array}{l}\text { Incerteza, surpresa, } \\
\text { adaptação. }\end{array}$ & $\begin{array}{l}\text { Adaptar as } \\
\text { necessidades do } \\
\text { projeto }\end{array}$ & $\begin{array}{l}\text { Adaptável para tipos } \\
\text { de produtos e } \\
\text { indústrias; universal: } \\
\text { todas as } \\
\text { organizações. }\end{array}$ \\
\hline Integrativo & $\begin{array}{l}\text { Incerteza, surpresa, } \\
\text { adaptação. }\end{array}$ & $\begin{array}{l}\text { Integrar componentes } \\
\text { múltiplos do processo } \\
\text { de desenvolvimento. }\end{array}$ & $\begin{array}{l}\text { Adaptável para tipos } \\
\text { de produtos, } \\
\text { organizações e } \\
\text { indústrias. }\end{array}$ \\
\hline Improvisacional & Complexidade & $\begin{array}{l}\text { Administrar eficiência } \\
\text { e flexibilidade ao } \\
\text { mesmo tempo. }\end{array}$ & $\begin{array}{l}\text { Dependente do } \\
\text { projeto particular. }\end{array}$ \\
\hline
\end{tabular}

Fonte: Adaptado de Cunha e Gomes (2003)

No modelo flexível a capacidade de introduzir mudanças no projeto em resposta a um ambiente em mudança com quase nenhuma penalidade, transforma-se em uma característica importante de modelos da inovação de produto quando o grau de agitação aumenta. Quando a flexibilidade é baixa, o custo econômico de modificar o produto é elevado. A necessidade de fazer coisas certas na primeira vez é o desafio principal quando o ambiente está permanentemente em mudança. É baseado no princípio de aprender fazendo e no fato de que o processo de inovação é um processo dinâmico. Este modelo é verificado em ambientes de negócio que são imprevisíveis, rápidos e povoados por concorrentes agressivos (por exemplo, indústria de computadores e softwares, multimídia e a indústria de moda). Aqui, as companhias com um modelo flexível podem 
continuar a incorporar novas informações do mercado no desenvolvimento do produto novo até quase o final, neutralizando assim as possíveis vantagens da concorrência.

O modelo integrativo verifica a qualidade da coordenação e do trabalho colaborativo entre todas as entidades envolvidas em desenvolvimento de produto. $\mathrm{O}$ modelo reconhece que o desenvolvimento é uma atividade complexa que requer a habilidade de obter, transformar e interpretar quantidades grandes de informação de várias áreas, marketing, equipe técnica, financeira, etc., a fim desenvolver idéias do produto e avaliar sua potencialidade econômica. Reforça o deslocamento da estrutura para os processos e desloca a importância das funções para o conhecimento. Outra implicação é que o conceito da integração estende agora à integração da inovação de produto em outros sistemas organizacionais tais como o sistema do negócio. Neste modelo o desenvolvimento é uma tarefa coletiva, que significa que as equipes e o teamwork são cruciais. As equipes de desenvolvimento são grupos de indivíduos interdependentes e envolvidos diretamente com um projeto. Estas equipes são criadas para trabalhar em um projeto particular e são dissolvidas no final dela. Às equipes podem ser dadas a autonomia e a responsabilidade para decidir um projeto.

O modelo improvisacional trabalha com a convergência temporal do planejamento e execução, o que significa que uma ação é improvisada quando constitui uma resposta deliberada para um problema ou oportunidade e é executada com os recursos disponíveis. No caso do desenvolvimento esta abordagem tenta facilitar a inovação sob condições impróprias. Este modelo pode ser mais bem usado em ambientes turbulentos. Combina elementos do modelo flexível com os elementos de abordagens tradicionais incluindo a necessidade de usar modelos de desenvolvimento como dispositivos do controle. Entretanto, o controle é executado com o uso de estruturas mínimas, que faz uma diferença significativa.

Fuller (1994) também apresenta um modelo de desenvolvimento de produtos (Figura 1) que possui como ponto de partida o estabelecimento dos objetivos mercadológicos para a empresa, que busca nas necessidades do consumidor oportunidades de mercado para o desenvolvimento de novos produtos. Por meio de algumas ferramentas se faz a geração de idéias de um novo produto.

O próximo passo desse modelo é o de agrupar todas as idéias para a sua posterior seleção. Esse processo de exclusão deve levar em conta alguns critérios como estudos de viabilidade técnica e financeira do produto e do processo. O produto a ser desenvolvido deve estar em sintonia com as necessidades do mercado.

Quando as idéias passam por esse filtro é escolhida a que mais se adequou aos critérios de seleção. A próxima etapa é para a parte técnica do desenvolvimento seguindo as recomendações relatadas pela equipe de desenvolvimento, onde são desenvolvidos protótipos dos produtos. Estes serão submetidos a uma avaliação, empregando os mesmos critérios da primeira seleção. Neste 
momento ocorre a primeira retroalimentação de informações do modelo. As informações são recolhidas e novamente serão analisadas pela equipe de desenvolvimento de produtos.

Em paralelo ao processo do desenvolvimento do protótipo inicia-se uma série de atividades que envolverão os departamentos financeiro, marketing e manufatura, cujas atividades estarão diretamente relacionadas a uma análise do plano de negócio desenvolvido pelo departamento financeiro. O departamento de manufatura determinará os caminhos a serem percorridos para facilitar a produção, mediando os requisitos de mão-de-obra e equipamentos.

Figura 1 - Modelo de desenvolvimento de produtos de Fuller

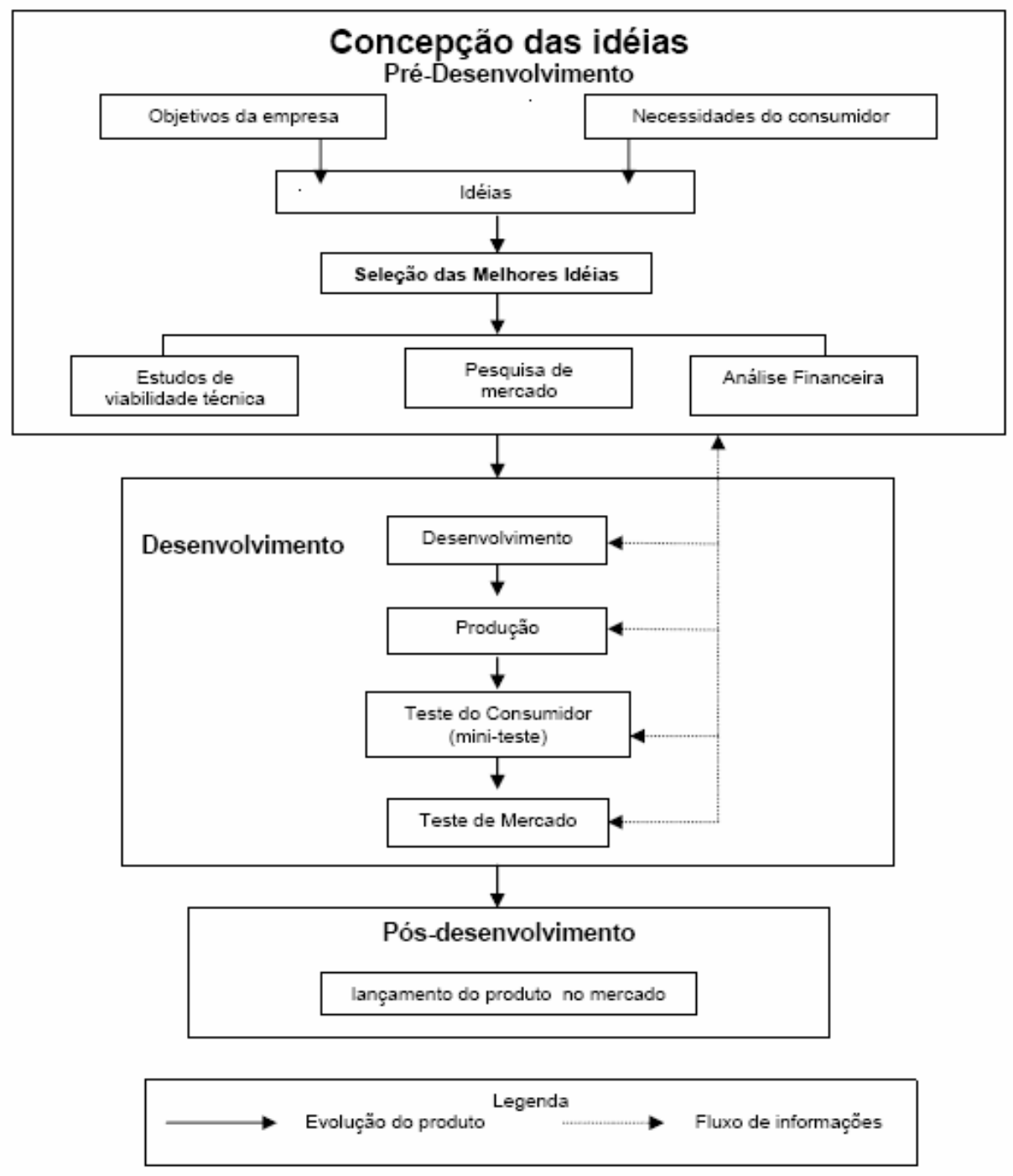

Fonte - Adaptado de Fuller ( 1994)

$\mathrm{Na}$ etapa de produção é desenvolvido um lote piloto e iniciam-se os testes do produto. $\mathrm{O}$ primeiro teste é feito dentro da empresa com um grupo de consumidores para avaliar o novo produto antes do seu lançamento. Os resultados podem gerar um refinamento do novo produto tanto na sua parte técnica como no processo. O outro teste é o teste de mercado onde uma região 
geográfica é escolhida sendo ofertado o produto e analisado o comportamento do mercado em relação a ele. A última etapa é o pós-desenvolvimento que é o lançamento do produto no mercado.

\section{Estudo de Caso}

Para a realização do estudo de caso foi escolhida uma empresa de médio porte do setor de cosméticos do estado do Rio de Janeiro que fabrica produtos para cabelo, maquilagem e perfumaria. O estudo foi desenvolvido ao longo do ano de 2004, tendo sido realizadas visitas e entrevistas com o objetivo de responder as seguintes questões: Como o desenvolvimento de novos produtos é inserido na estratégia da empresa; quais as etapas que compõem o processo de desenvolvimento de produtos; e se existe um processo estruturado para tal, isto é, segue algum determinado modelo de processo de desenvolvimento de produto encontrado na literatura específica sobre o assunto.

Verifica-se que, de acordo com Mintzberg e Quinn (2001), a empresa utiliza desde uma estratégia simples de extensão do produto onde existe um acréscimo às linhas de produtos da empresa até uma estratégia de proliferação da linha do produto onde esta busca uma diferenciação no mercado através do lançamento de um produto novo ou então de um novo design de embalagem.

Observa-se que, conforme Baxter (2000), a empresa concentra-se mais nas estratégias defensivas e tradicionais dependendo da linha de produto. Ao optar por uma estratégia de relançamento de produtos a empresa adota uma estratégia tradicional, pois esta se limita a mudanças mínimas no produto. Já nas estratégias de lançamento simples e composto é utilizado o conceito de estratégia defensiva, pois a empresa deseja seguir as tendências do mercado, porém sem arcar com custos muito altos de desenvolvimento do produto. Assim utiliza-se de embalagens já padronizadas da empresa ao lançar estes produtos.

Observa-se, portanto que as estratégias adotadas pela empresa procuram minimizar os riscos e custos inerentes à criação de novos mercados bem como a necessidade de investimentos em pesquisa e desenvolvimento para a introdução de inovações radicais em seus produtos.

As decisões quanto ao desenvolvimento de produtos estão de acordo com a estratégia da empresa e este processo abrange de uma forma geral, as fases de concepção de idéias, desenvolvimento ou detalhamento do projeto do produto até o lançamento do mesmo no mercado. $\mathrm{Na}$ empresa percebe-se que o fluxograma descrevendo o processo de desenvolvimento do produto, se apresenta de forma estruturada, com o fluxo de informações seguindo os modelos referenciados (Figura 2).

A diferença do modelo da empresa para o modelo de Fuller (1994) é quanto à realização de testes de mercado. O autor salienta a importância destes testes ao longo do processo de desenvolvimento do produto a fim de reduzir riscos à medida que o projeto vai evoluindo. Já o fato 
da empresa não realizar testes de mercado propicia a ela uma maior agilidade do seu processo, porém podendo gerar incertezas quanto ao seu sucesso no mercado. Este fato pode ser explicado em função do tipo de estratégia adotada pela empresa que, conforme explicado anteriormente, envolve menores riscos.

No modelo seqüencial de Cunha e Gomes (2003) a equipe de desenvolvimento de produtos deve primeiro finalizar uma etapa para depois passar para a seguinte. Na empresa encontram-se esta estratégia no início do processo quando está em definição qual produto que irá se produzir.

Figura 2 - Fluxograma de desenvolvimento de produtos da empresa

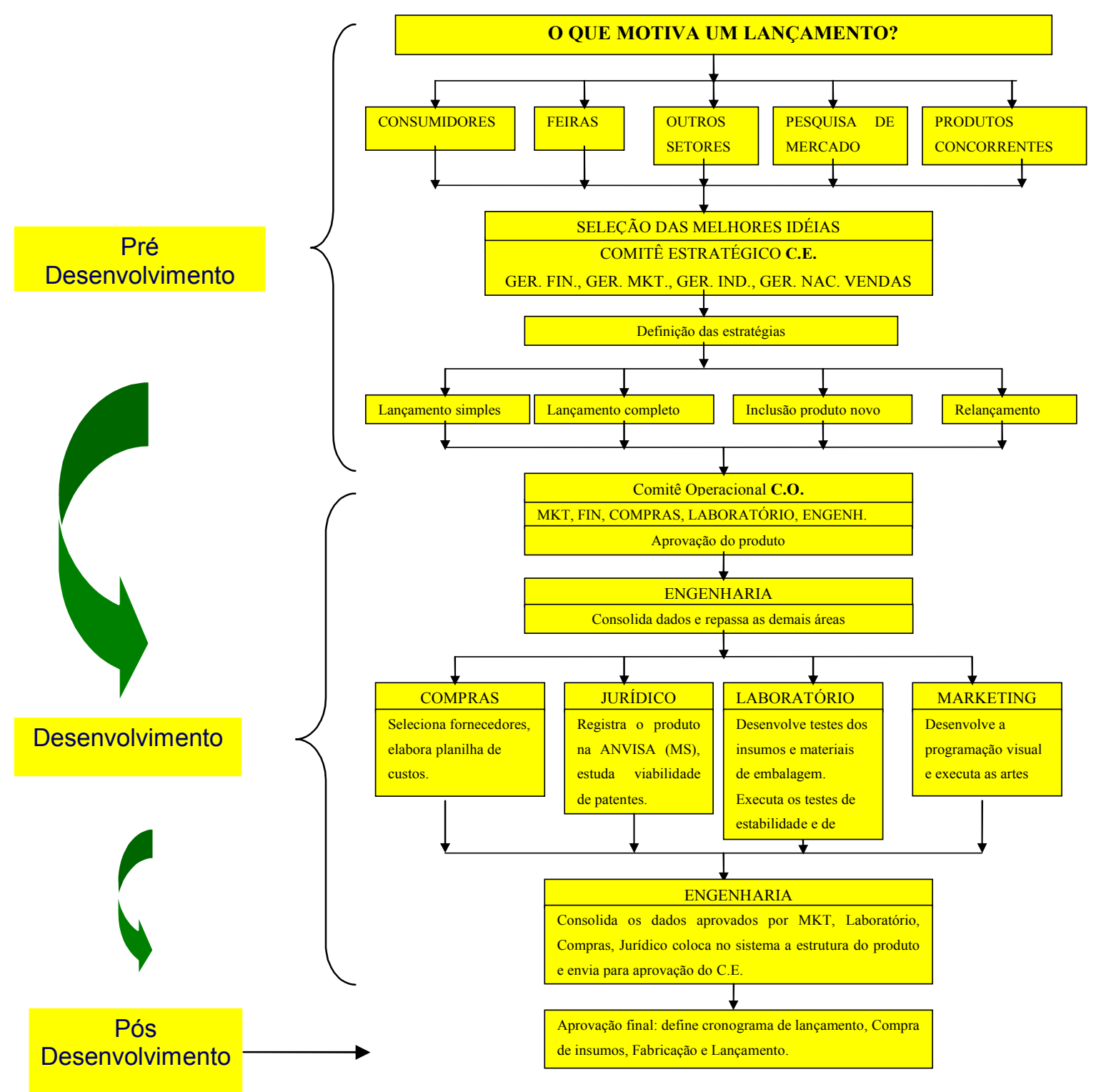

Fonte: Avelar (2005) 
Dependendo de qual estratégia a empresa adota, verifica-se na fase de desenvolvimento do produto a utilização do modelo de compressão já que algumas etapas podem ser suprimidas ou não dependendo da necessidade.

O modelo flexível pode ser verificado durante o processo de desenvolvimento quando mudanças acontecem e as pessoas envolvidas desenvolvem a capacidade de se adaptar e reformular em tempo hábil o produto para que prazos não sejam estourados ou o lançamento do produto comprometido.

\title{
5. Conclusão
}

O presente trabalho procurou mostrar o processo de desenvolvimento de produtos em uma empresa de médio porte do setor de cosméticos tendo como objetivo responder às três perguntas inicialmente formuladas com relação à empresa.

De acordo com os resultados obtidos, verificou-se que a empresa está próxima aos processos atuais de desenvolvimento, considerando-se o referencial teórico utilizado. Respondendo às perguntas formuladas, a empresa pode ser classificada como uma empresa que busca a sua inserção nas cadeias produtivas globais e que apesar de não ser de grande porte, desempenha papel importante com lançamentos de conceitos novos no mercado e não só a simples reprodução do que é desenvolvido pelas grandes empresas. Apresenta quatro tipos de estratégias que vão desde o lançamento de um produto novo até o relançamento de produtos da empresa variando entre as estratégias o grau de risco desejado pela empresa no processo.

O processo de desenvolvimento de produtos está estruturado com suas etapas bem definidas. Verifica-se que a empresa não segue um modelo específico de desenvolvimento, devido a alguns fatores como as singularidades do setor, ao fato de ser uma empresa de médio porte competindo com grandes empresas transnacionais e devido à definição da estratégia a ser adotada no processo. No caso da empresa, não existe um melhor modelo e sim vários modelos que podem ser adaptados à empresa dependendo do tipo de produto a ser desenvolvido.

\begin{abstract}
It can be said that amongst the four variables related to the development - land, capital, work and technology -, technology is the one that more weight has in the determination of a sector competitive capacity. Currently with the leveling of the companies to the aspects of quality and productivity, the technology management started to blunt as important competitiveness differential. The importance of the technological innovation for the competitiveness is unquestioned; either to gain markets through the introduction of new products and processes or to produce with maximum physical exploitation those variables in order to reduce costs. Inside this context, the development of new processes and products is becoming a primordial function for the companies. The cosmetic industry has presented a very special growth in the last years with an increase in its invoicing of
\end{abstract}


$19 \%$ in 2004 in comparison to 2003. This sector growth reflects an incited competitiveness demanding the companies the constant launching of new products as well as a reformulation of old packings. To follow this dynamism the adoption of strategies directed toward the management of innovation and product process development becomes necessary. The objective of the work is to present a case study in a company of the sector in order to verify how is the product development process in relation to the existing models in literature. These models possess as starting point the establishment of marketing objectives for the company, who searches in the consumer necessities and market chances the focus for new products development.

Key-words: technological innovation; cosmetic industry; new products development

\section{Referências}

AVElAR, A. C. M. - Desenvolvimento de Produtos na Indústria Nacional de Cosméticos: Um Estudo de Caso. Dissertação de Mestrado em Tecnologia - Rio de Janeiro: Centro Federal de Educação Tecnológica - CEFET-RJ, 2005.

BAXTER, M. - Projeto de Produto: Guia prático para design de novos produtos, $2^{a}$ ed., Edgard Blücher, São Paulo, 2000 .

CLARK, K.B.; FUJIMOTO, T. - Product development performance: strategy, organization, and management in the word auto industry, Boston-Mass, HBS Press, 1991.

CUNHA, M.P.; GOMES, J.F.S. - "Order and Disorder in Product Innovation Models", Creativity and Innovation Management, v. 12, n. 3, 2003.

cross ${ }^{\text {ref }}$

FLEURY, A. - “Gerenciamento do Desenvolvimento de Produtos na Economia Globalizada”. In: Anais do I Congresso Brasileiro de Gestão do Desenvolvimento de Produto, Minas Gerais, 1999.

FULLER, G.W. - New Food Product Development: From Concept Marketplace, CRC Press LLC, Florida, 1994.

GARCIA, R.; FURTADO, J. - Estudo da competitividade de cadeias integradas no Brasil: impacto das zonas de livre comércio - cadeia: cosméticos, Núcleo de Economia Industrial e da Tecnologia (UNICAMP-IENEIT), 2002.

MARINHO, V.M.C. - "Como as empresas brasileiras de cosméticos estão utilizando o conhecimento tradicional e as plantas medicinais”. In: Anais do XXVI Reunião Anual sobre Evolução, Sistemática e Ecologia Micromoleculares Instituto de Química - XXVI RESEM, Rio de Janeiro, 2004.

MINTZBERG, H; QUINN, J.B. - O Processo da Estratégia, 3ª ed., Bookmann, Porto Alegre, 2001.

MONTANA, J. - "Innovación; El reto empresarial del siglo XXI”. In: GÜELL, A.M.; VILA, M. (Orgs), El arte de innovar en la empresa, Ediciones Del Bronce, 2001.

\section{Dados completos do primeiro autor:}

Nome completo: Ana Cristina Maia Avelar

Filiação Institucional: CEFET - RJ

Endereço completo para correspondência : Rua São Francisco Xavier, n 478 apt 1401

Maracanã - Rio de Janeiro - RJ - Brasil - CEP - 20550-013

Telefones para contato: (21) 2254-4676

e-mail: anacrisavelar@yahoo.com.Br

Recebido para publicação em: 14/01/2006 Aceito para publicação em: 16/04/2006 
